\title{
Evaluation of biological activity of the conjugates of granulocyte-macrophage colony stimulating factorwith alendronic acid
}

\author{
Volosnikova Ekaterina Aleksandrovna \\ department of technology development \\ and pilot production of biologicals \\ IMBT FBRI SRC VB «Vector», \\ Rospotrebnadzor, Berdsk, Russia \\ volosnikova_ea@vector.nsc.ru \\ Shimina Galina Grigor'evna \\ department of biological studies \\ IMBT FBRI SRC VB «Vector», \\ Rospotrebnadzor, Berdsk, Russia
}

\author{
Esina Tat'yana Igorevna \\ department of technology development \\ and pilot production of biologicals \\ IMBT FBRI SRC VB «Vector», \\ Rospotrebnadzor, Berdsk, Russia
}

Danilenko Elena Dmitrievna department of biological studies IMBT FBRI SRC VB «Vector», Rospotrebnadzor, Berdsk, Russia

\author{
Bateneva Alena Vladimirovna \\ department of biological studies \\ IMBT FBRI SRC VB «Vector», \\ Rospotrebnadzor, Berdsk, Russia
}

\begin{abstract}
We have developed a method for the synthesis of rhGM-CSF protein conjugates with alendronic acid by use of water-soluble carbodiimide. The biological activity and accumulation in bone marrow of GM-CSF conjugates with ALN have been confirmed.
\end{abstract}

Keywords - Recombinant human granulocyte-macrophage colony stimulating factor, neutropenia, affinity, activity, conjugate

\section{Introduction}

Recombinant human granulocyte-macrophage colony stimulating factor (GM-CSF) is used as a hematopoietic stimulant in patients with various diseases accompanied by neutropenia. However, the clinical use of GM-CSF preparations revealed a number of adverse reactions associated with its long-term use (fever, myalgia, bone pain, etc.). The development of targeted therapeutics is one of the ways to reduce the administered dose of the drug, and, as a consequence, its toxicity. Targeted drug delivery to the bone marrow, apparently, can enhance the growth and differentiation of hematopoietic cells due to GM-CSF localization at the site of hematopoiesis. As a vector molecule, alendronic acid (ALN), having affinity for the bone tissue, can be used.

\section{Materials and Methods}

For this study we used reagents from AppliChem (Germany), Sigma-Aldrich (USA), Bio-Rad (USA). For the synthesis of conjugates we used the preparation of GM-CSF (substance) manufactured by IMBT FBRI SRC VB "Vector".

The molecular weight of the conjugates was determined by use of vertical gel electrophoresis in $15 \%$ polyacrylamide gel under denaturing conditions, with Coomassie R-250 staining. The study of the conjugates ability to accumulate in bone tissue was performed on an in vitro bone matrix model by use of hydroxyapatite chromatography. The in vitro biological activity of GM-CSF within the conjugates was evaluated by the stimulation level of proliferation of cytokinedependent human erythroleukemia cells (TF-1). The in vivo hemostimulating activity was determined by use of a model of cytostatic myelosuppression induced by cyclophosphamide administration into mice. The level of distribution and accumulation of GM-CSF preparations in blood, femoral tissue, and bone marrow was performed on CD-1 outbred mice (ICR) with a single intravenous administration at the effective dose of $90 \mu \mathrm{g} / \mathrm{kg}$. The content of rhGM-CSF in the samples taken 3 minutes, 1, 4 and 24 hours post administration was determined by the enzyme immunoassay with "Human Granulocyte-Macrophage Colony Stimulating Factor (GMCSF) ELISA Kit” (Cusabio, China).

\section{Results}

The synthesis of GM-CSF with ALN was carried out through the carboxyl group of the protein with crosslinking agent 1-ethyl-3- [3-dimethylaminopropyl] carbodiimide (EDC). The conjugation was carried out on a solid phase, for what a chromatographic column with hydroxyapatite (HAP) was used. During the synthesis, the reaction components: GM-CSF, EDC, ALN were subsequently loaded onto a HAP column balanced with $2 \mathrm{mM}$ potassium phosphate buffer, $\mathrm{pH}$ 7.0. Conjugates were eluted with $0.2 \mathrm{M}$ potassium phosphate buffer, $\mathrm{pH}$ 7.0. The resulting preparation of GM-CSF conjugates was sterilely poured and frozen at $-20{ }^{\circ} \mathrm{C}$.

It has been determined that the molecular weight of the resulting conjugate was $15.4 \pm 0.3 \mathrm{kDa}$, which corresponds to the molecular weight of GM-CSF and, therefore, indicates the monomeric form of the protein within the conjugate. The protein, within the conjugate with ALN, retained the ability to stimulate the proliferation of human erythroleukemia cells TF-1: the ED50 of the conjugate was $0.38 \mathrm{ng} / \mathrm{ml}$ and did not differ much from the ED50 of GM-CSF substance $(0.28 \mathrm{ng} /$ $\mathrm{ml}$ ). The in vitro experiment confirmed a high affinity of the conjugate for hydroxyapatite, used as a bone matrix analogue.

On the model of cytostatic myelosuppression, it was shown that GM-CSF conjugate, as well as the initial protein, accelerated the restoration of bone marrow cellularity and the number of segmentonuclear neutrophils in peripheral blood. The stimulating effect of the conjugate on the total number of karyocytes was more pronounced.

A study of distribution and accumulation of GM-CSF conjugates in the organs and tissues of mice confirmed their increased ability to penetrate into the bone tissue and bone marrow. Thus, the protein content in the femur during 4 hours after the conjugate administration was 2.9-3.9 times higher than the values recorded after the administration of GM-CSF 
substance (Fig. 1a). The values of the indicator in this group remained higher compared to the control level (intact animals) until the end of the first day. The level of GM-CSF in bone marrow cells 3 minutes after the conjugate administration significantly exceeded the control indicator by 161 times, while after the substance administration, only 67 times (Fig. $1 b)$.

The conjugated GM-CSF circulated longer in the peripheral blood of mice. Thus, 3 minutes post administration, the protein content in the blood was $19.2 \%$ of the administered dose for the conjugate and $7.2 \%$ for the substance; statistically significant differences between the groups persisted for at least 4 hours (Fig. 1c). It is important to note that GM-CSF conjugate was not detected in the blood by the end of the first day after the administration.

\section{Conclusion}

The data obtained indicate that conjugation of GM-CSF with ALN has enhanced the accumulation of GM-CSF in the bone marrow and increased its level of hemostimulating activity. Therefore, the developed approach is a promising platform for the further development of drugs with increased tropism to the bone marrow and hemostimulant effect.

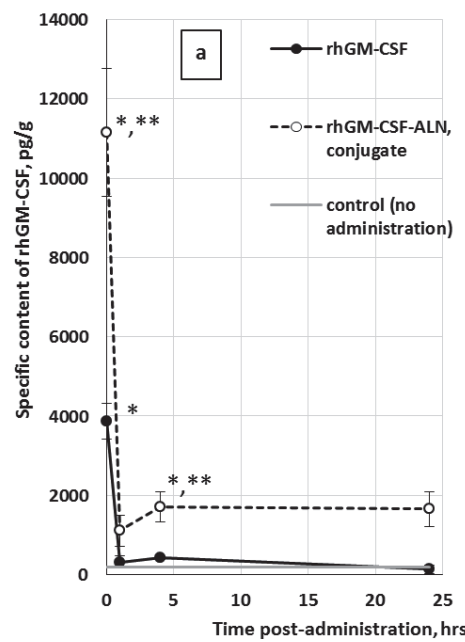

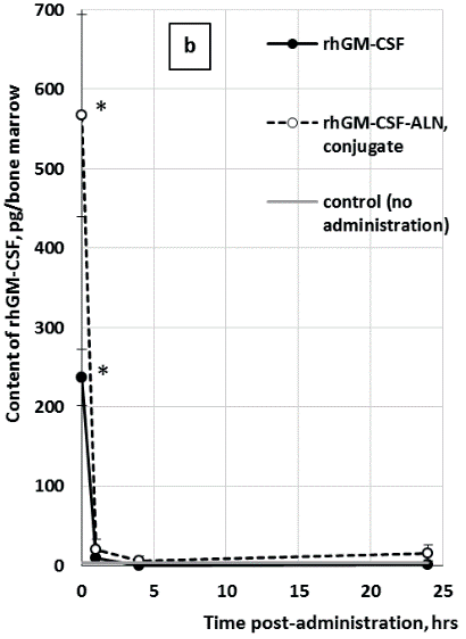

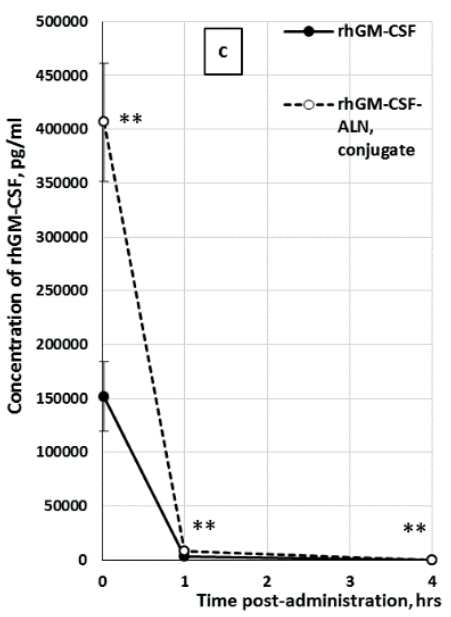

Fig. 1. Dynamics of changes of GM-CSF content in the femurs (a), bone marrow (b) and blood serum (c) of mice after a single intravenous administration of GM-CSF preparations. The significance of intergroup differences was assessed by the nonparametric MannWhitney U-test at a significance level of $p<0.0170$ for tissues, $p$ $<0.05$ for blood; * - differences are statistically significant compared to the control; ** - differences are statistically significant compared to GM-CSF substance.

\section{ACKNOWLEDGMENT}

This work was financially supported by the Russian Foundation for Basic Research, a project in the framework of the contest "My First Grant", application No. 18-315$00236 \backslash 19$. 